\title{
The CPD Project
}

This link of traditional paper publishing with

electronic access is an

exciting precursor to

the opportunities that

will be available

in the future
$\mathbf{T}$ The April edition of Primary Dental Care contains the pilot for a new joint initiative between the British Dental Association (BDA) and the Faculty of General Dental Practitioners (UK) (FGDP). This initiative will look at the viability of providing accreditation for continuing professional development (CPD) using the medium of a dental journal.

Thus the CPD initiative will examine the possibility of using a question and answer self-assessment 'quiz' within the pages of both professional journals over a six month period, from July to December this year. Readers will be invited to complete the questions and submit their answers for marking, and will then be provided with appropriate feedback.

In order to refine the whole process, Primary Dental Care (the journal of the FGDP) published a pilot set of questions this month to assess readers' reactions. This pilot will then form a basis for the final sections of the questions for both Primary Dental Care and the $B D J$ from July to December.

The questions for the self-assessment quiz will focus on endodontics (for the first three months) and cross infection control (for the second half of the period). All questions will be based around the Department of Health CAL programmes, Endodontic Diagnosis and Management (CAL 4) and SafeQuest: A look at cross infection control for the dental team (CAL 12). Aside from focusing the learning experience for those taking part in this initiative, this move will help those practitioners who normally find it difficult to attend postgraduate meetings.

The pilot is funded by the National Centre for Continuing Professional Education of Dentists (NCCPED) and will be available for all dentists to participate in. Those who are not members of either the BDA or the FGDP can obtain the appropriate pages from the respective journals by contacting the BDA or by visiting the $B D J$ or Faculty web-sites as described below. Successful candidates will receive accreditation and a certificate from the Faculty of General Dental Practitioners.

The intention of the scheme is not to replace the numerous programmes of postgraduate education currently being held in postgraduate centres, but to complement all existing forms of learning. The advantage of this type of programme is its flexibility, allowing practitioners to carry out study and response to the self-assessment at times that suit them.

Further details will be available on the $B D J$ and Faculty web sites at: http://www.bdj.co.uk and http://www.rcseng.ac.uk/fgdp respectively. Practitioners wishing to obtain the accreditation who are not members of the BDA or the Faculty of General Dental Practitioners will be able to obtain the information by contacting the $B D J$ (details will be available in the journals of both organisations and in this month's issue of Dental Practice.)

This link of traditional paper publishing with electronic access (through both the CAL programmes and the internet) is an exciting precursor to the opportunities that will be available in the future. As internet access increases in popularity (as will happen as soon as the cable and satellite TV companies align with computer companies to produce the TV/internet hardware in every home), so opportunities to revolutionise postgraduate learning (as opposed to postgraduate education) will arise. We cannot understand how this will change our perception and experience of learning yet, in the same way that ancient scholars could not foresee the effect of printing on them. All we can be sure of is that it will be completely different from the way we use educational resources at present. 